\title{
ON A MULTI-CLASS QUEUE WITH \\ CLASS DEPENDENT WINDOW-FLOW CONTROL
}

\author{
by \\ Raif O. Onvural \\ H.G. Perros \\ Computer Science Department \\ Center for Communications and Signal Processing \\ North Carolina State University \\ Raleigh, NC, 27695-8206, USA
}

and

U. Koerner

Department of Communication Systems

Lund University

Box 118

S-22100 Lund, SWEDEN

CCSP-TR-88/17

\begin{abstract}
We consider a single server queue shared by $\mathrm{N}$ different classes of jobs. Class $i$ jobs arrive at the queue in a Poisson fashion with a class dependent rate. The total number of class $i$ jobs allowed to wait in the queue is $w_{i}$, the window size of this class. A class $i$ job that arrives to find $w_{i}$ class $i$ jobs in the shared queue is forced to wait in an input queue. There is one input queue for each class. All classes receive the same service at the shared queue, which is exponentially distributed. We analyze the two-class model numerically. For more than two classes, we obtain an approximate decomposition algorithm that utilizes the two-class numerical procedure. Validation tests showed that the approximation procedure is fairly accurate.
\end{abstract}

* Supported by a grant from AIRMICS through the Center for Communications and Signal Processing, NC State University 


\section{Introduction}

One of the resource management tasks which critically affects the performance of computer rommunication networks is the flow control. i.e. the regulation of accepted traffic such that the network is not overloaded. Unless proper How control is enacted, throughput of the network may significantly degrade below its optimum value. The principle of flow control is to shift congestion from the interior of the network to the points of admittance. The most widely known protocol to control the flow in a network is the window-flow control. The idea is simply to refuse admittance of new traffic when a certain number of messages in a region of the network is unacknowledged. The permissible upper bound of unacknowledged messages is called the window size (cf. Reiser [6]).

In this paper, we consider the case where multiple connections use the same resource, depicted by a single server queue. The traffic along each connection is regulated with a window-flow mechanism. In general, most of the studies reported in the literature on window-flow control deal with a single connection (see Fdida, Perros and Wilk [1] for a literature review). Reiser [5] modeled a computer communication network consisting of many virtual routes with end-to-end window flow control as a closed multi-chain queueing network. Each chain represented a different virtual route under the assumption of a loss system. That is, he assumed that packets that arrive to find a full window are discarded. He proposed a computationally efficient approximation procedure based on mean value analysis for evaluating large closed multi chain networks. The model is used 
to obtain transit delays and blocking probabilities. Lam [2] extended the class of multi-chain queueing networks of the product form type to include mechanisms of state dependent lost and triggered arrivals.

The problem of analyzing multiple window control mechanisms can be formulated as a single or multi-class closed queueing network with population constraints. The population constraint is imposed as follows: In the single class case, it is assumed that a subnetwork is subject to a population constraint. That is, only upto a predefined number of customers are allowed in the subnetwork. The remaining customers are queued in an input queue. In the multi-class case, for a particular subnetwork, each class (or a group of classes) has its own population constraint. These models have been analyzed approximately as closed queueing networks by several authors. A review of these approximations can be found in Thomasian and Bay [7]. The reader is also referred to Krzesinski and Teunissen [2] for a more recent approximation algorithm. We note that these models were originally developed for multiprogramming systems. They may not be suitable for modeling window-flow control mechanisms, which naturally give rise to open queueing networks. It is necessary, therefore, to develop open multi-class queueing networks with population constraints.

In this paper, we study a queueing model consisting of a single server multiclass queue as shown in figure 1 (hereafter referred to as the shared queue). The total number of class $\mathrm{i}$ jobs allowed to wait in this queue may not exceed a finite number, $w_{i}$ (hereafter referred to as the window size), $i=1 \ldots \mathrm{N}$, where $\mathrm{N}$ is the 
number of classes. A class $i$ job that arrives to the network at a time that there are $u$ : jobs in the shared queue is torced to wait in an input queue. Let $C$ : be the capacity of the input queue. $C_{\text {, }}$ can be finite or infinite. $A$ class $i$ job waiting in the input queue is allowed to enter the shared queue only when a class $i$ job departs from the network. There is one input queue per class.

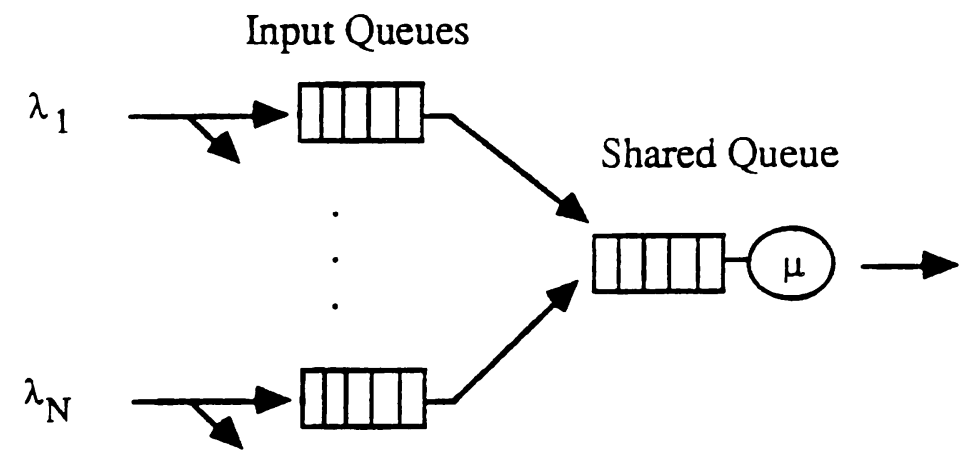

Figure 1: A multi-class open queueing model

Let $\lambda_{i}, \mu$ be respectively the rate arrivals of class $i$ jobs and the mean service time of a job in the shared queue. All interarrival times and service times are assumed to be exponentially distributed. Jobs at each input queue move into the shared queue in a FEO manner. The service discipline at the shared queue is assumed to be processor sharing. We note that the marginal probabilities of each class are the same whether the service discipline at the shared queue is processor sharing, service in random order, or FIFO. This can be easily verified by comparing the steady-state equations for each discipline. 
Exact analysis of this model is rather difficult. In this paper, we analyze this queueing model numerically and approximately. In particular. the two-class model is analyzed numerically. For more than two classes. we analyze the model using an approximate decomposition algorithm which utilizes the two-class numerical procedure. In the following section, we describe the numerical procedure. In section III, we present the approximation procedure and its validation. In section $\Gamma$, we analyze the behavior of some performance metrics with respect to the arrival rates and window sizes. Finally, the conclusions are given in section V.

\section{The Queueing Model with Two Classes}

Let us consider the queueing model described in section I with two classes. Let $\left(n_{1}, n_{2}\right)$ denote its state where $n_{i}$ is the number of class $i$ jobs in the network, $\mathrm{i}=1,2$. We have $0 \leq n_{i} \leq w_{i}+C_{i}$. Furthermore, let $P\left(n_{1}, n_{2}\right)$ be the steady-state probability of being in state $\left(n_{1}, n_{2}\right)$. Seeing that the system under study is Markovian we have the following steady-state equations:

for $w_{1} \leq n_{1} \leq C_{1}+w_{1}, w_{2} \leq n_{2} \leq C_{2}+w_{1}$

$$
\begin{aligned}
\left(\lambda_{1}+\lambda_{2}+\mu\right) P\left(n_{1}, n_{2}\right)= & \lambda_{1} P\left(n_{1}-1, n_{2}\right)+\lambda_{2} P\left(n_{1}, n_{2}-1\right)+ \\
& \frac{w_{1}}{w_{1}+w_{2}} \mu P\left(n_{1}+1, n_{2}\right)+\frac{w_{2}}{w_{1}+w_{2}} \mu P\left(n_{1}, n_{2}+1\right)
\end{aligned}
$$


for $0 \leq n_{1}<w_{1}, w_{2} \leq n_{2} \leq C_{2}+w_{2}$

$$
\begin{aligned}
\left(\lambda_{1}+\lambda_{2}+\mu\right) P\left(n_{1}, n_{2}\right)= & \lambda_{1} P\left(n_{1}-1 . n_{2}\right)+\lambda_{2} P\left(n_{1}, n_{2}-1\right)+ \\
& \frac{n_{1}+1}{n_{1}+w_{2}+1} \mu P\left(n_{1}+1 . n_{2}\right)+\frac{\omega_{2}}{n_{1}+w_{2}} \mu P\left(n_{1}, n_{2}+1\right)
\end{aligned}
$$

for $w_{1} \leq n_{1} \leq C_{1}+w_{1}, 0 \leq n_{2}<w_{2}$

$$
\begin{aligned}
\left(\lambda_{1}+\lambda_{2}+\mu\right) P\left(n_{1}, n_{2}\right)= & \lambda_{1} P\left(n_{1}-1, n_{2}\right)+\lambda_{2} P\left(n_{1}, n_{2}-1\right)+ \\
& \frac{w_{1}}{w_{1}+n_{2}} \mu P\left(n_{1}+1, n_{2}\right)+\frac{n_{2}+1}{w_{1}+n_{2}+1} \mu P\left(n_{1}, n_{2}+1\right)
\end{aligned}
$$

for $0 \leq n_{1}<w_{1}, 0 \leq n_{2}<w_{2}$, and $n_{1}+n_{2}>0$

$$
\begin{aligned}
\left(\lambda_{1}+\lambda_{2}+\mu\right) P\left(n_{1}, n_{2}\right)= & \lambda_{1} P\left(n_{1}-1, n_{2}\right)+\lambda_{2} P\left(n_{1}, n_{2}-1\right)+ \\
& \frac{n_{1}+1}{n_{1}+n_{2}+1} \mu P\left(n_{1}+1, n_{2}\right)+\frac{n_{2}+1}{n_{1}+n_{2}+1} \mu P\left(n_{1}, n_{2}+1\right)
\end{aligned}
$$

and,

$$
\left(\lambda_{1}+\lambda_{2}\right) P(0,0)=\mu(P(1,0)+P(0,1))
$$

We note that in these equations, the probability of being in an infeasible state, i.e. $n_{i}>C_{i}+w_{i}$, or $n_{i}<0$, is equal to zero.

The above system of equations does not possess a product form solution. Furthermore, it is very difficult to obtain an exact analytic solution. In view of this, we analyze this model numerically. This involves the following three-step procedure: i) Generation of states; ii) Creating the rate matrix, $Q$ and iii) Numerical solution of the system $Q^{T} P\left(n_{1}, n_{2}\right)=0$. The states can be generated easily as 
follows:

$$
\begin{aligned}
& \text { for } i:=0 \text { to }\left(w_{1}+C_{1}\right) \text { do } \\
& \text { for } j:=0 \text { to }\left(w_{2}+C_{2}\right) \text { do } \\
& \text { begin } \\
& \text { ind }:=\text { ind }+1 ; \\
& \text { states }(\text { ind }):=(i, j) \\
& \text { end } ;
\end{aligned}
$$

The generation of the rate matrix can be accomplished easily by going through the list of states and generating all feasible transitions for each state. In general, from an arbitrary state $\left(n_{1}, n_{2}\right)$ there are four possible transitions to states $\left(n_{1}+1, n_{2}\right),\left(n_{1}, n_{2}+1\right),\left(n_{1}-1, n_{2}\right)$, and $\left(n_{1}, n_{2}-1\right)$. The order of the rate matrix is:

$$
\left(C_{1}+w_{1}+1\right) *\left(C_{2}+w_{2}+1\right)
$$

Once the rate matrix is set up, the steady-state queue length distribution can be obtained using a numerical technique such as power method, Gauss-Seidel method, etc.

A numerical procedure using the power method was implemented on a VAX-11/785 to calculate the joint queue length distributions for up to 4 classes. As expected, the CPU time required to generate the states and to generate the rate matrix was observed to be negligible. However, the numerical solution of the system $Q^{T} \mathrm{P}\left(n_{1}, n_{2}\right)=0$ required a considerable amount of CPU time even for the two-class case. Clearly, the numerical approach becomes infeasible as the number of classes increases (see section III.3). 


\section{The Approximation Procedure}

In this section. we present a decomposition procedure for the approximate analysis of the $\mathrm{N}$-class queueing system shown in Figure 1 . Below, we first consider the case of finite capacity input queues, i.e., $C_{i}<x, i=1, \ldots, N$. The algorithm is then extended to the case of infinite capacity input queues in section IiI.2. Finally, in section III.3, both cases are validated and the time complexity of the algorithm is given.

\section{1. Finite Capacity Input Queues}

Let us consider the $\mathrm{N}$-class queueing model shown in Figure 1 with parameters $\lambda_{i}, w_{i}, \mu$ and $C_{i}<x, \mathrm{i}=1, \ldots, \mathrm{N}$, with $\mathrm{N} \geq 3$. We decompose the N-class system into $\mathrm{N}$ two-class systems, the first class of which is the class under consideration and the second class is the aggregation of all other classes.

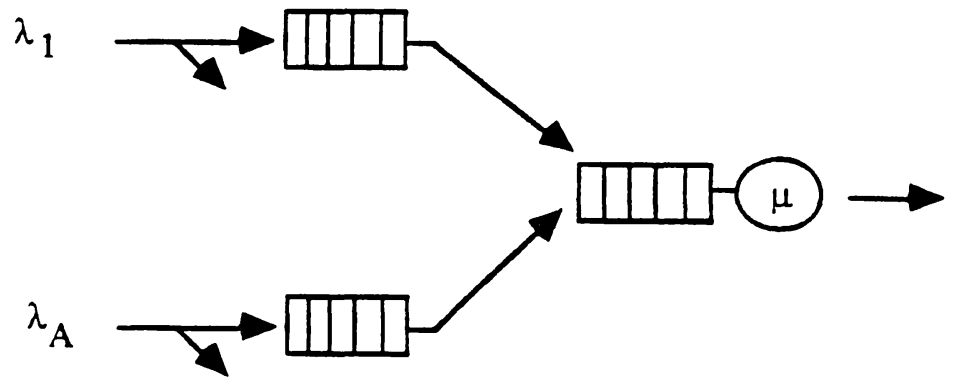

Figure 2: A two-class model

Without loss of generality, consider class 1 as the class under consideration. The second class is then the aggregation of classes 2 to $\mathrm{N}$ (hereafter referred to as class A) as shown in figure 2. The accuracy of the approximation clearly depends on how well the parameters of the aggregate class are specified. Let $\lambda_{A}, w_{A}$ and 
$C_{A}$ be respectively the arrival rate. window size and capacity of the input queue of the aggregated class. In the original model. the total number of class $A$ jobs in the shared queue varies between zero and the sum of the window capacities of classes 2 to $\perp$. Therefore, set

$$
w_{A}=\sum_{i=2}^{v} w_{i}
$$

We also set the arrival rate of class $A$ jobs equal to the sum of the arrival rates of the individual classes, i.e.

$$
\lambda_{A}=\sum_{i=2}^{N} \lambda_{i}
$$

Setting these values for $\lambda_{A}$ and $w_{A}$ as shown above results in an overestimation of the number of class $\mathrm{A}$ jobs in the shared queue. The total arrival rate is equal to $\lambda_{A}=\sum_{i=2}^{N} \lambda_{i}$ as long as the total number of class $\mathrm{A}$ jobs in the shared queue is less than $\min _{j=2, \ldots, N} w_{j}$. Once this limit is reached, then in the real system, jobs belonging to the class with the smallest window size may not enter the shared queue. Clearly, this is not considered in the aggregate class with $\lambda_{A}=\sum_{i=2}^{N} \lambda_{i}$ and $w_{A}=\sum_{i=2}^{N} w_{i}$. We reduce the effect of overestimating the number of class A jobs in the shared queue by fixing appropriately the capacity of the input queue of the aggregate class. The size of this input queue can be anything between zero and $\sum_{i=2}^{N} C_{i}$. For a given arrival rate $\lambda_{A}$, the probability of having $\mathrm{k}$ jobs in the shared queue increases as $C_{A}$ increases where $0 \leq \mathrm{k} \leq w_{A}$. Therefore, in order to counter 
balance the overestimation of the shared queue. $C_{1}$ should be small. Accordingly, we studied the following problem numerically: Ciiven the parameters of class 1 . $\lambda_{1}=\sum_{i=2}^{v} \lambda_{l}$. and $u_{1}=\sum_{i=2}^{Y} w_{i}$ what is the value of $c_{1}$ such that the marginal probabilities of class 1 jobs are approximated with minimum error. Rather surprisingly, this numerical study suggested that $C_{4}=0,1$ or 2 . Although these values of $C_{A}$ do not always give the best approximate values, they are observed to be the best in most cases and they are never too far from the value of $C_{A}$ which results in the best approximate values. This is true for a range of different values of the parameters of the queueing model.

Once we fix the parameters of the aggregate class, the resulting two-class model is solved numerically using the procedure given in section II. The steps of the algorithm are summarized as follows:

Step 1: Without loss of generality, consider class 1 as the class under consideration. Let the parameters of the aggregate class be as follows: $w_{A}=\sum_{i=2}^{N} w_{i}$, $\lambda_{A}=\sum_{i=2}^{N} \lambda_{i}, \rho=\sum_{i=1}^{N} \lambda_{i} / \mu$ and $C_{A}= \begin{cases}2 & \text { if } \rho<0.8 \\ 1 & \text { if } 0.8 \leq \rho\end{cases}$

Step 2: Solve the two class network numerically to calculate the marginal queue length distribution of class 1.

By renumbering classes, the algorithm is readily applicable to any other class. 


\section{2. Infinite Capacity Input Queues}

We will now consider the $\vee$-class model with $C_{2}=x, i=1 \ldots \ldots . N$, and $\quad i \geq 3$. Let $\pi(n)$ be the total number of jobs in the network without distinguishing between classes. Then, the following lemma is immediate by appealing directly to the steady-state equations.

Lemma: Consider an $\mathrm{N}$-class queueing model as shown in figure 1 with parameters $\lambda_{i}, w_{i}, C_{i}, i=1, \ldots, N$, and $\mu$. Let $C_{i}=x$ for all $\mathrm{i}$. Furthermore, let $\pi(\mathrm{n})$ be the probability that total number of jobs in the network is $n$. Then

$$
\pi(n-1) \sum_{i=1}^{N} \lambda_{i}=\mu \pi(n)
$$

where, $\pi(0)=1-\left(\sum_{i=1}^{N} \lambda_{i} / \mu\right),\left(\sum_{i=1}^{N} \lambda_{i} / \mu\right)<1$.

Hence, the system behaves like an $M / M / 1$ queue if we do not distinguish between classes. In order to use the numerical procedure developed in section II, we should know the total capacity for each class, i.e. $C_{i}+w_{i}$. In the case of infinite capacity input queues, we obtain these values by first determining the total finite capacity, $C^{*}$, such that $\sum_{n=0}^{C^{*}} \pi(n)$ is approximately equal to 1 , where $\sum_{n=0}^{C^{*}} \pi(n)=1-\rho^{C^{*}+1}$. In particular, we use the following procedure to calculate $C^{*}$.

Step 1: Let $\rho=\sum_{i=1}^{N} \lambda_{i} / \mu$ with $\rho<1$, and let $C^{*}=1$.

Step 2: while 1-p $\rho^{C^{*}+1}<0.99999$ do

$$
C^{*}=C^{*}+1
$$


Once, $C^{*}$ is calculated, we apply the algorithm presented above for finite capacity input queues assuming $C^{\prime}:=C^{\prime \prime}$ tor all $\mathrm{i}$.

III. 3. Time Complexity and Accuracy of the Procedure

The approximation procedure presented above decomposes the $\mathrm{N}$-class queueing system into $\mathrm{N}$ two-class systems. The number of states in each two-class system is $B_{i}=\left(C_{i}+w_{i}\right)\left(2+w_{A}\right)$. Hence, the total time complexity of the algorithm to calculate the marginal probabilities of all classes is $O\left(\prod_{i=1}^{N} B_{i}^{3}\right)$. We note that the time complexity of the exact numerical procedure is $O\left(\left(\prod_{i=1}^{N}\left(C_{i}+w_{i}\right)\right)^{3}\right)$. Hence, the approximation is considerably faster than solving the entire queueing system numerically. In table 1 , we give the CPU times of the exact numerical procedure and the approximation procedure for 9 different examples. The algorithm was applied to the $\mathrm{N}$-class model, where the number of classes was varied from 3 to 4 . The validation tests show that the relative error percentage (i.e. 100* (exact value-approximate value)/exact value) was fairly low. The numerical results were obtained using the numerical procedure described in section II. A representative set of examples are given in figures 12 to 20 . The parameters of these examples are summarized in table 2 . 


\begin{tabular}{|c|l|c|c|c|c|c|}
\hline$N$ & $\lambda_{1}, . ., \lambda_{N}$ & $\mu$ & $C_{1}, \ldots, C_{V}$ & $w_{1} \ldots, u_{N}$ & $\begin{array}{c}\text { Numerical } \\
\text { Proc. (sec) }\end{array}$ & $\begin{array}{c}\text { App. } \\
\text { Proc. (sec) }\end{array}$ \\
\hline \hline 3 & $(1.2 .3)$ & 12 & $(x, x, x), 9$ & $(3.2 .4)$ & 73.3 & 9.6 \\
3 & $(1,3,2)$ & 9 & $(x, x, x), 11$ & $(2.3,4)$ & 389.9 & 19.2 \\
3 & $(1,1.1)$ & $j$ & $(4.4,4)$ & $(2.2,2)$ & 6.1 & 5.3 \\
3 & $(1,1,1)$ & $j$ & $(8.8,8)$ & $(2,2,2)$ & 33.4 & 9.1 \\
3 & $(1,2,3)$ & 7 & $(8,8,8)$ & $(2,2,2)$ & 120.2 & 12.5 \\
3 & $(1,2,3)$ & 12 & $(x, x, x), 8$ & $(3.3,3)$ & 73.3 & 11.4 \\
3 & $(1,1,1)$ & 7 & $(x, x, x), 9$ & $(2,2,2)$ & 113.2 & 6.3 \\
4 & $(1,2,5,4)$ & 18 & $(6,6,6,6)$ & $(2,2,2,2)$ & 719.5 & 12.1 \\
4 & $(1,2,1,4)$ & 15 & $(6,6,6,6)$ & $(2,3,5,4)$ & 656.4 & 21.1 \\
\hline
\end{tabular}

Table 1: CPU times of the exact numerical procedure and the approximation procedure

\section{Performance Metrics}

The performance metrics used in this analysis are defined as follows: Let $\pi_{i}\left(n_{i}\right)$ be the marginal probability of having $n_{i}$ class $\mathrm{i}$ jobs in the network, $M Q L-S_{i}$ be the mean number of class $\mathrm{i}$ jobs in the shared queue, where

$$
M Q L-S_{i}=\sum_{n_{i}=1}^{C_{i}+w_{i}} \min \left(n_{i}, w_{i}\right) \pi_{i}\left(n_{i}\right)
$$

$M Q L-I_{i}$ be the mean number of class $\mathrm{i}$ jobs in the input queue, where $M Q L-I_{i}$

$=\sum_{n_{i}=w_{i}+1}^{C_{i}}\left(n_{i}-w_{i}\right) \pi_{i}\left(n_{i}\right)$, and $T_{i}$ be the throughput of class $i$ jobs, where

$$
T_{i}=\left(\begin{array}{ll}
\lambda_{i} & \text { if } C_{i}=\infty \\
\lambda_{i}\left(1-\pi_{i}\left(C_{i}+w_{i}\right)\right) & \text { otherwise }
\end{array}\right.
$$

For the two-class model we study the behavior of $M Q L-S_{i}, M Q L-I_{i}$, and $T_{i}$ as i) the arrival rate of a class varies and ii) the window size of a class varies 
while all other parameters are kept the same. The values of these performance metrics are calculated numerically using the procedure described in section II.

Consider now the two-class model with parameters $C_{2}=11, \lambda_{1}=2 . \lambda_{2}=1$. $w_{2}=2$. and $\mu=4$. In figures 3 to $5, w_{1}$ is varied from 2 to 11 while $C_{1}=13-w_{1}$. We observe that both $M Q L-S_{1}$ and $M Q L-S_{2}$ increases as $w_{1}$ increases. As $w_{1}$ increases, there are more class 1 jobs in the shared queue. This in turn reduces the rate at which class 2 jobs depart from the shared queue, thus increasing $M Q L-S_{2}$. As $w_{1}$ increases, there are less class 1 jobs waiting in the input queue, hence $M Q L-I_{1}$ decreases. However, as the departure rate of class 2 jobs decreases, $M Q L-I_{2}$ increases seeing that more jobs arrive to find $w_{2}$ jobs in the shared queue. For this example, both $T_{i}$ 's stay about the same as $w_{1}$ increases. Seeing that their values are very close to their respective arrival rates, we conclude that the capacities of the input queues are as if they were infinite. We note that in this example $\lambda_{1}+\lambda_{2}<\mu$. Now, let us consider another example where $\lambda_{1}+\lambda_{2}>\mu$. Let $C_{2}=9, \lambda_{1}=2, \lambda_{2}=3, \mu=2$ and $w_{2}=4$. In figures 6 to $8, w_{1}$ is varied from 2 to 11 while $C_{1}=13-w_{1}$. In this case, all performance metrics are pushed to their limits, i.e. $M Q L-S_{i} \simeq w_{i}, T_{1}+T_{2} \simeq \mu$, and

$$
M Q L-I_{i}= \begin{cases}13-w_{1} & i=1 \\ 9 & i=2\end{cases}
$$

Finally, we give an example to show the effect of varying the mean arrival time of a class while all the other parameters are kept the same. In particular, consider the two-class model with parameters $C_{1}=11, C_{2}=13, w_{1}=4, w_{1}=2$, 
$\mu=7$. and $\lambda_{2}=1$. In figures 9 to $11 . \lambda_{1}$ is varied from 1 to 10 . Increasing $\lambda_{1}$ causes both $. M Q L-S_{1}$ and $. M Q L-I_{1}$ to increase as expected. As $M Q L-S_{1}$ increases. the departure rate of class 2 jobs decreases, causing $. M Q L-\Xi_{2}$ to increase slightly. This in turn causes $M Q L-I_{2}$ to increase.

\section{Conclusions}

We presented a model for the case where multiple connections use the same resource, depicted by a single server queue. A numerical procedure was implemented to calculate the steady-state joint queue length distributions. The number of states grows rapidly with the number of classes. In view of this, the numerical approach becomes infeasible even with a few classes. This necessitated the development of an approximation algorithm for more than two classes. The procedure decomposes the model to $\mathrm{N}$ two-class models, one for each class. Each two-class model is then analyzed numerically. Validation tests showed that the results are fairly accurate.

In real situations, more than one node may be required to represent a virtual route. Furthermore, although the approximate procedure saves considerable amount of CPU time in the case of more than two classes, an accurate approximation procedure for two-class model may result in even more savings. We are currently working on these two problems as an extension of the ideas presented in this paper. 


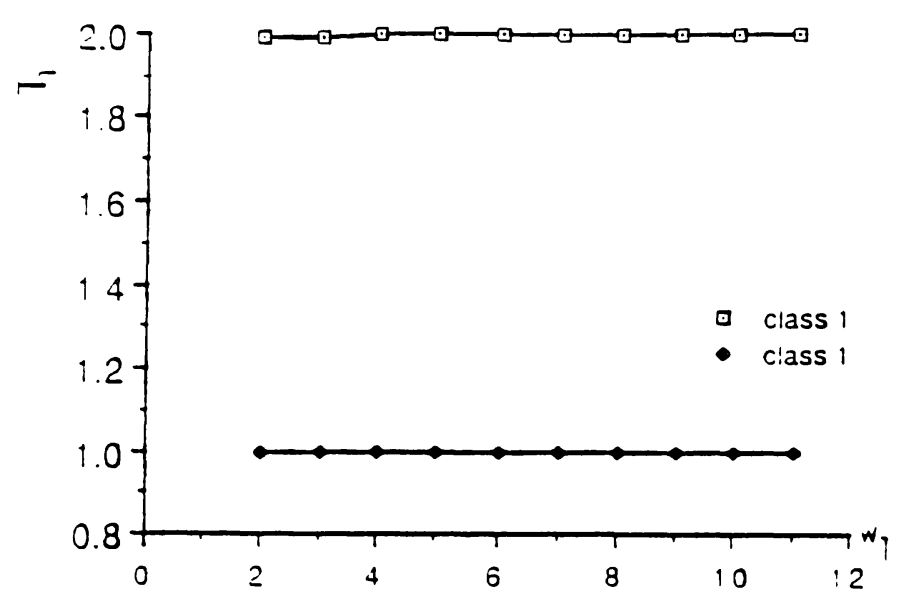

Fig 3: Throughput of class I jobs vs $w_{1}$

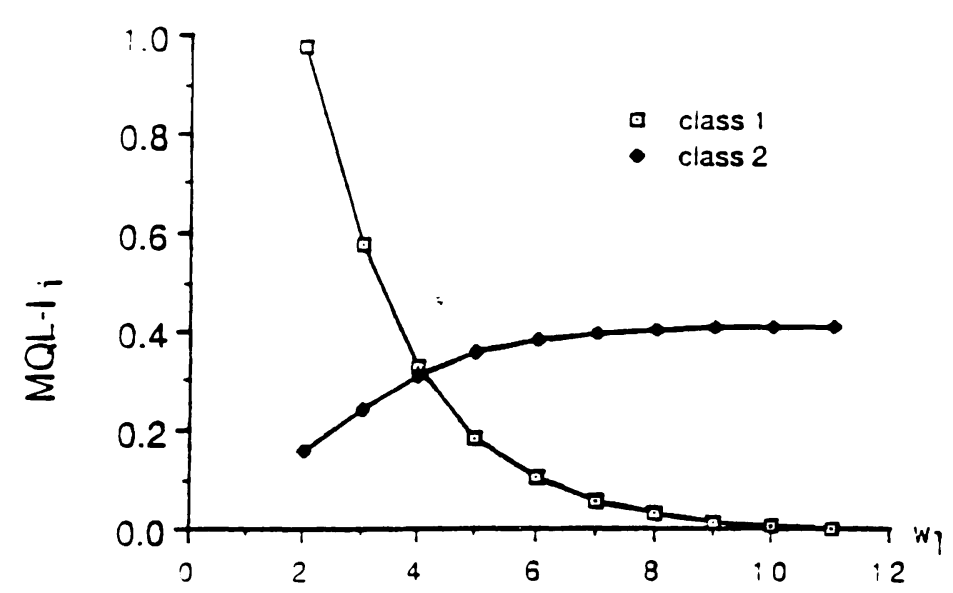

Fig 5:Mean no of class $i$ jobs in the input queue vs $w_{\text {, }}$

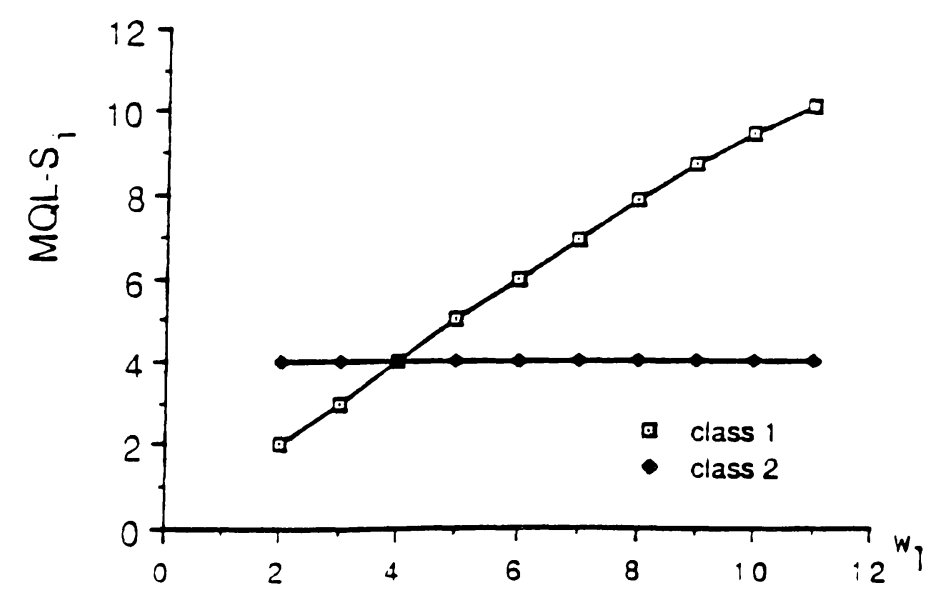

Fig 7:Mean no of class $i$ jobs in the shared queue vs $w_{1}$

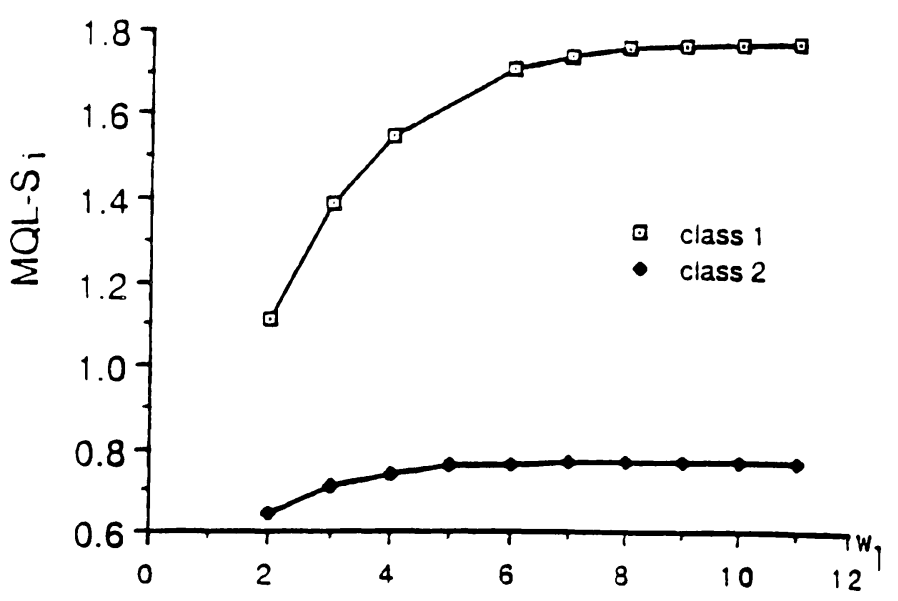

Fig 4:Mean no of class $i$ jobs in the shared queve vs w

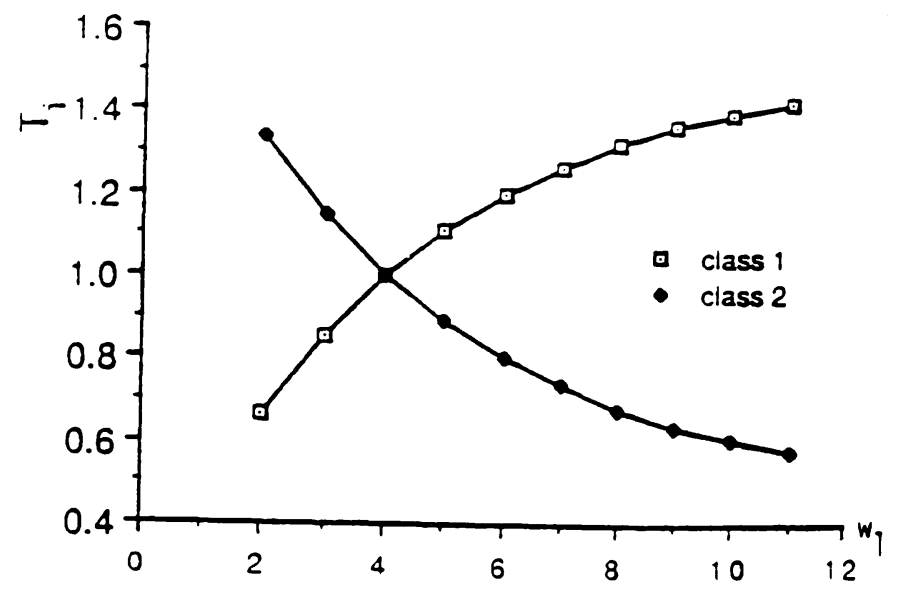

Fig 6: Throughput of class 1 jobs vs $w_{1}$

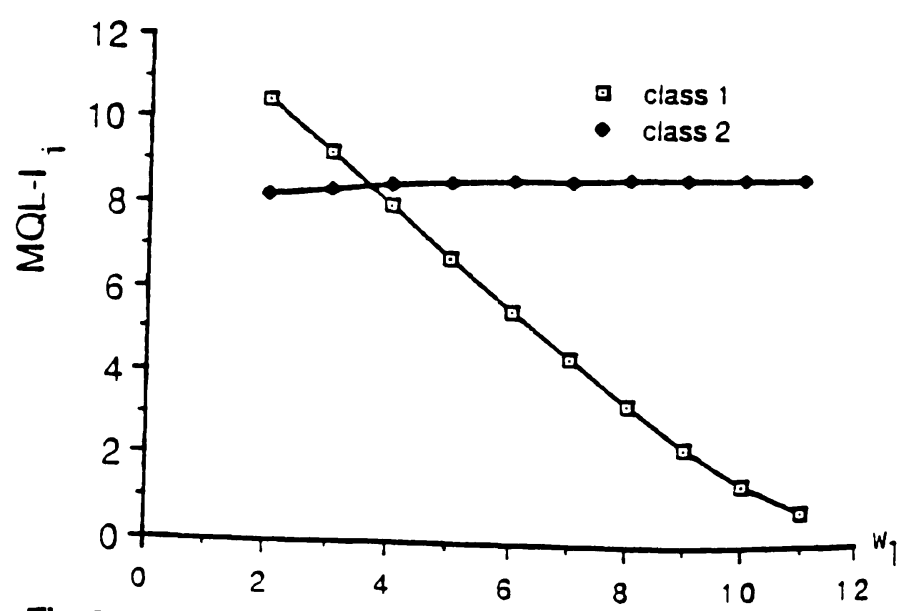

Fig 8:Mean no of class $i$ jobs in the input queue vs $w_{T}$ 


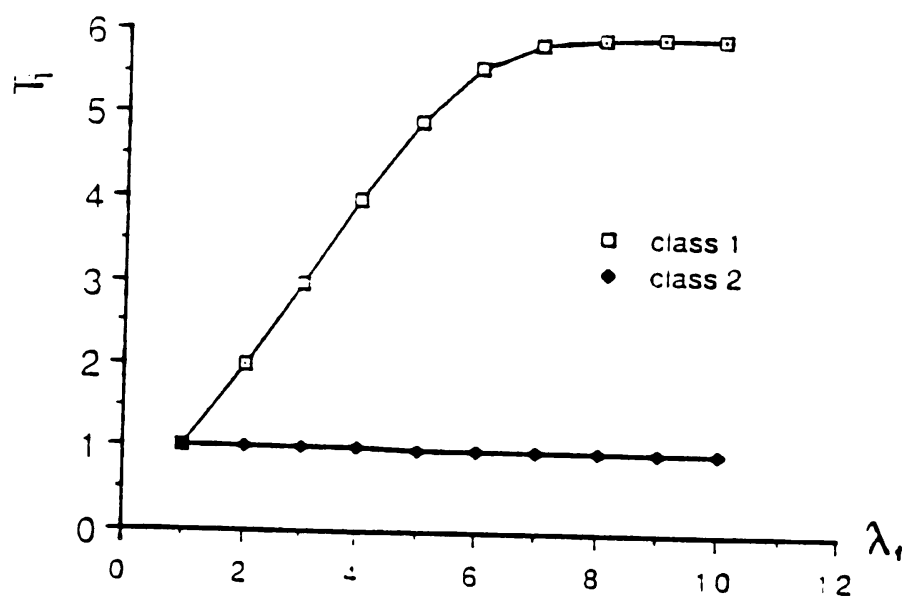

Fig 9:Througnput of class $i$ jobs vs $\lambda_{1}$

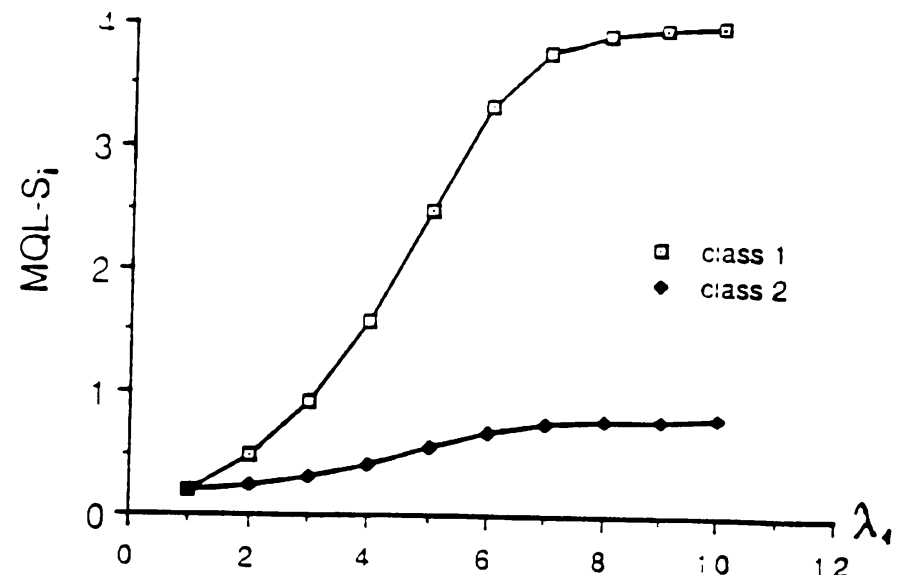

Fig 10:Mean no of class $i$ jobs in the snared queue vs $\lambda_{1}$

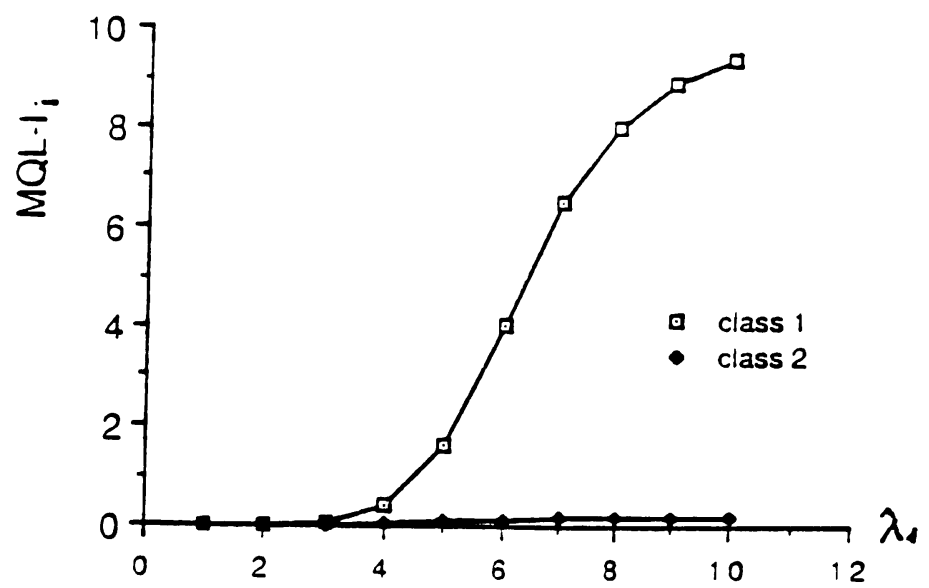

Fig 11:Mean no of class $\mathrm{i}$ jobs in the input queue vs $\lambda_{1}$

\begin{tabular}{|c||c|c|c|c||}
\hline \hline Ex \# & $\left(\lambda_{1}, \lambda_{2}, \lambda_{3}, \lambda_{4}\right)$ & $\left.C_{1} \cdot C_{2} \cdot C_{3}, C_{4}\right)$ & $\left(w_{1}, w_{2}, w_{3}, w_{4}\right)$ & $\mu$ \\
\hline 1 & $(1,2,5,4)$ & $(4,4,4,4)$ & $(2,2,2,2)$ & 18 \\
2 & $(1,2,1,4)$ & $(4,3,1,2)$ & $(2,3,5,4)$ & 15 \\
3 & $(1.2,3)$ & $(5,5,5)$ & $(3,3,3)$ & 12 \\
4 & $(3,2,3,4)$ & $(4.3,4.3)$ & $(2,3,2,3)$ & 16 \\
5 & $(3,2,4,1)$ & $(5,4,5,5)$ & $(2,3,2,2)$ & 7 \\
6 & $(3,2,4,1)$ & $(5,4,5,5)$ & $(2,3,2,2)$ & 10 \\
7 & $(3,2,3,4)$ & $(4,3,4,3)$ & $(2,3.2 .3)$ & 16 \\
8 & $(3,2.3,4)$ & $(4,3,4,3)$ & $(2,3,2.3)$ & 10 \\
9 & $(1.3,2)$ & $(9,8,7)$ & $(2,3.4)$ & 9 \\
\hline
\end{tabular}

Table 2: Data for the examples in figures 12 to 20 . 


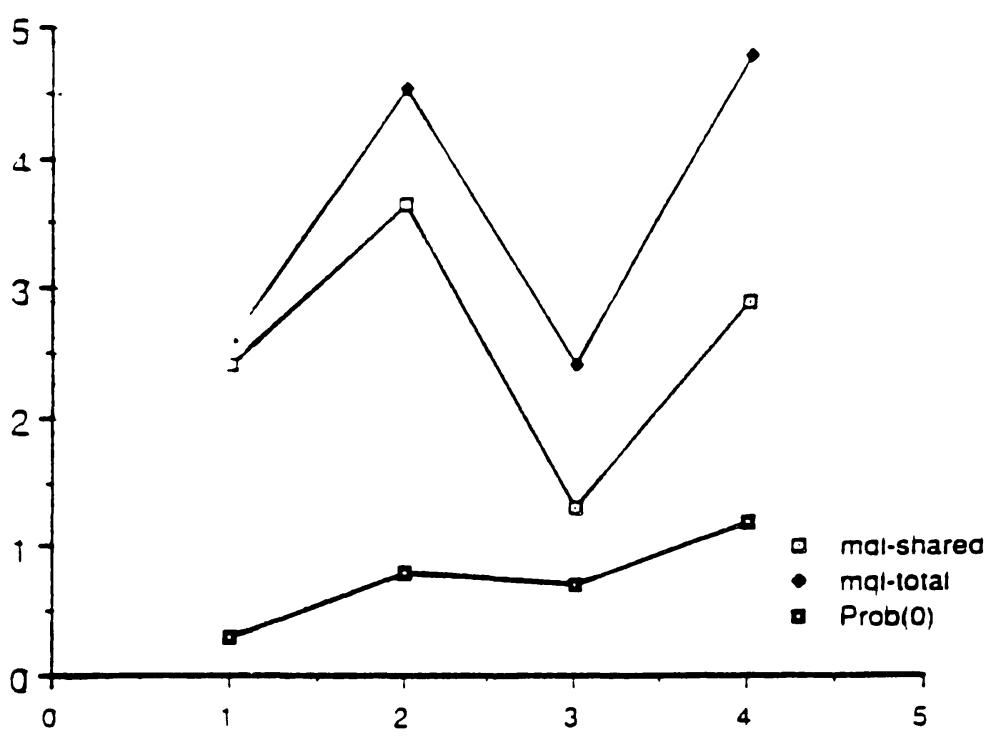

Class \#

$$
\begin{gathered}
F: g \text { 12: Relative error } \% \text { for each class } \\
\text { (Example I in Table 2) }
\end{gathered}
$$

0.707

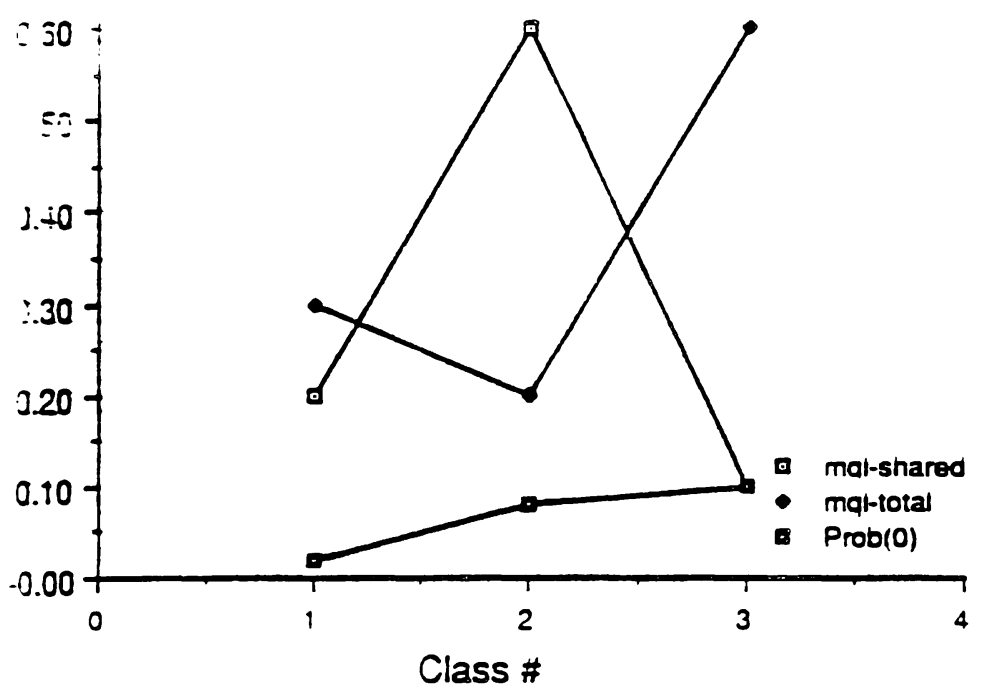

Fig 14:Relative error\% for each class (Example 3 in Table 2)

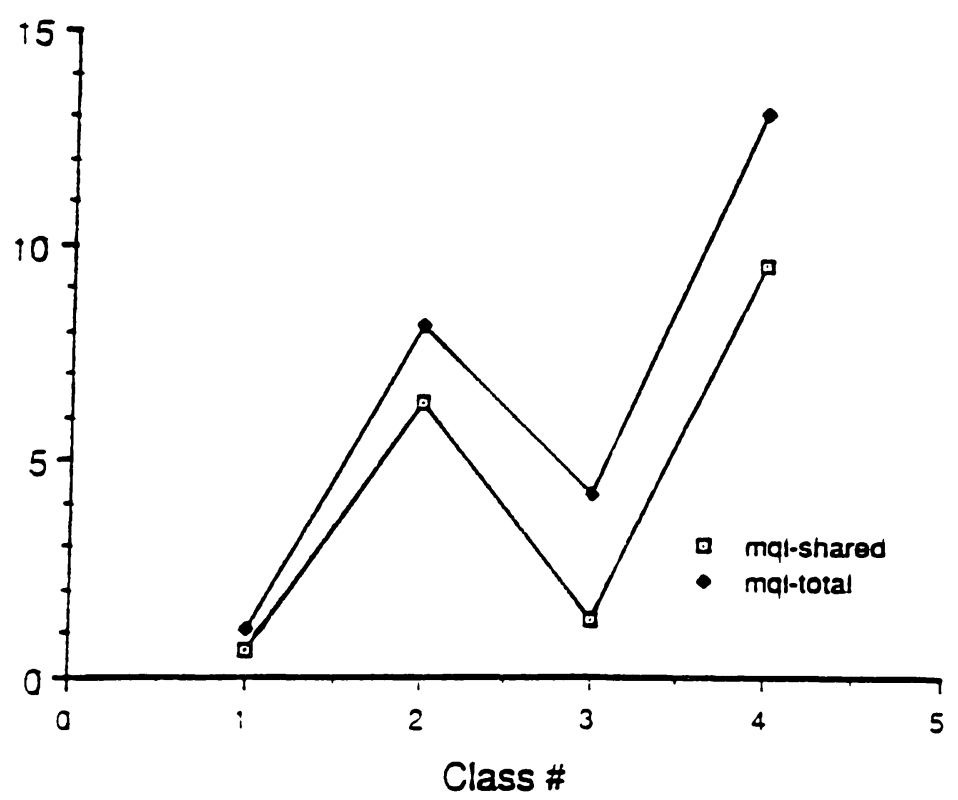

Fig 16: Relative error: for each class (Example 5 in Table 2)

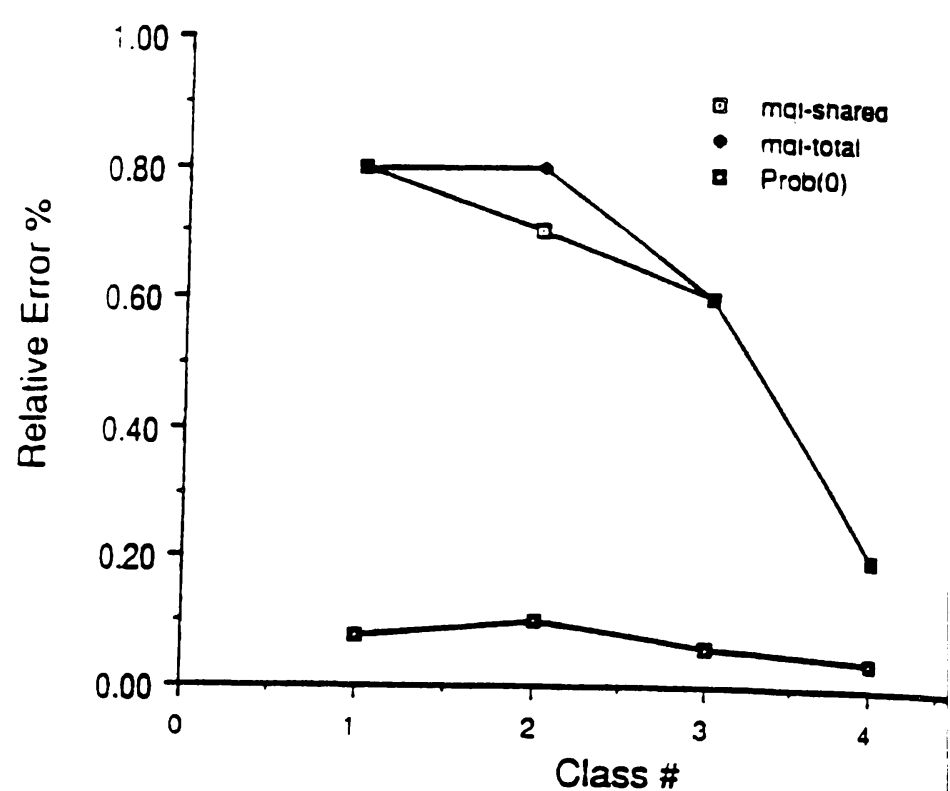

Fig 13: Relative error\% for each c'as (Example 2 in Table 2)

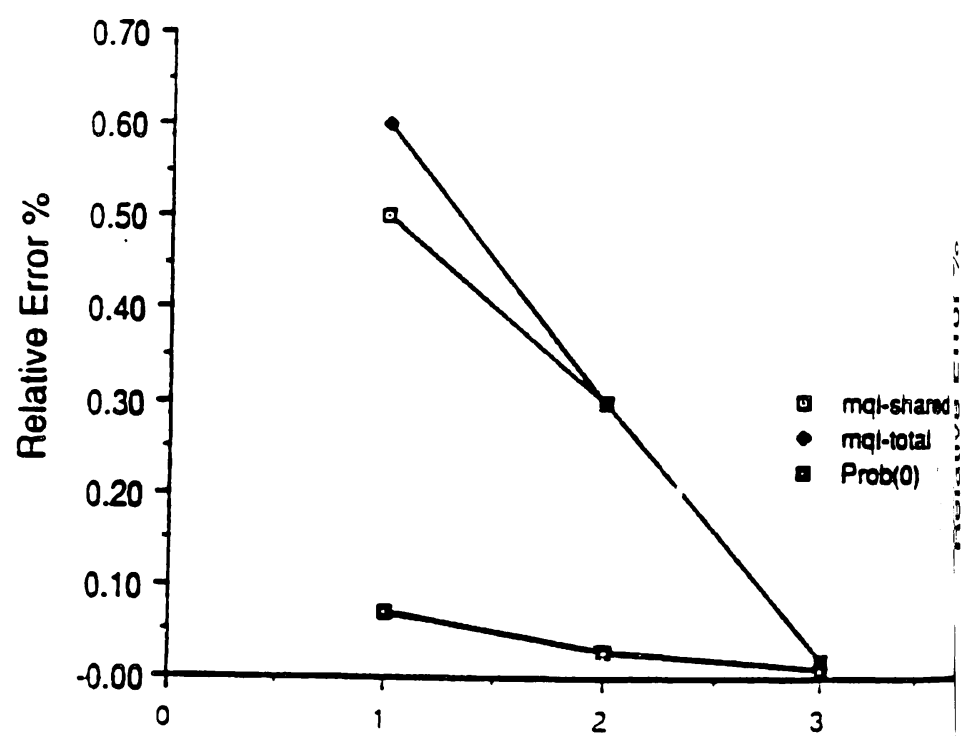

class \#

Fig 15: Relative error : for each class (Example 4 in Table 2)

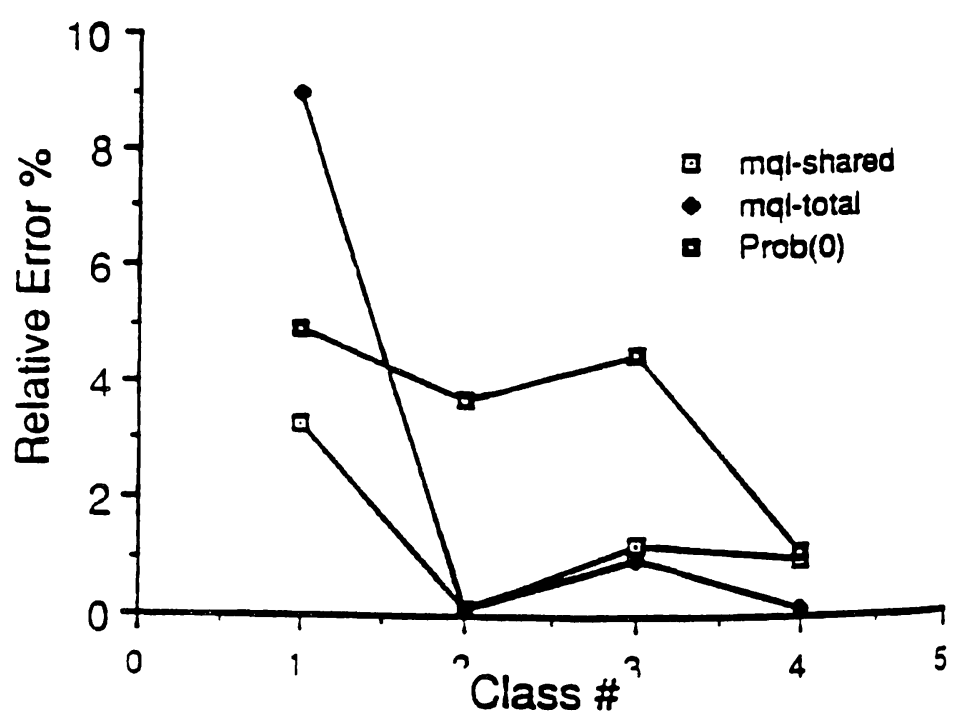

Fig 17: Relative error \% for each ol (Example 6 in Table 2) 


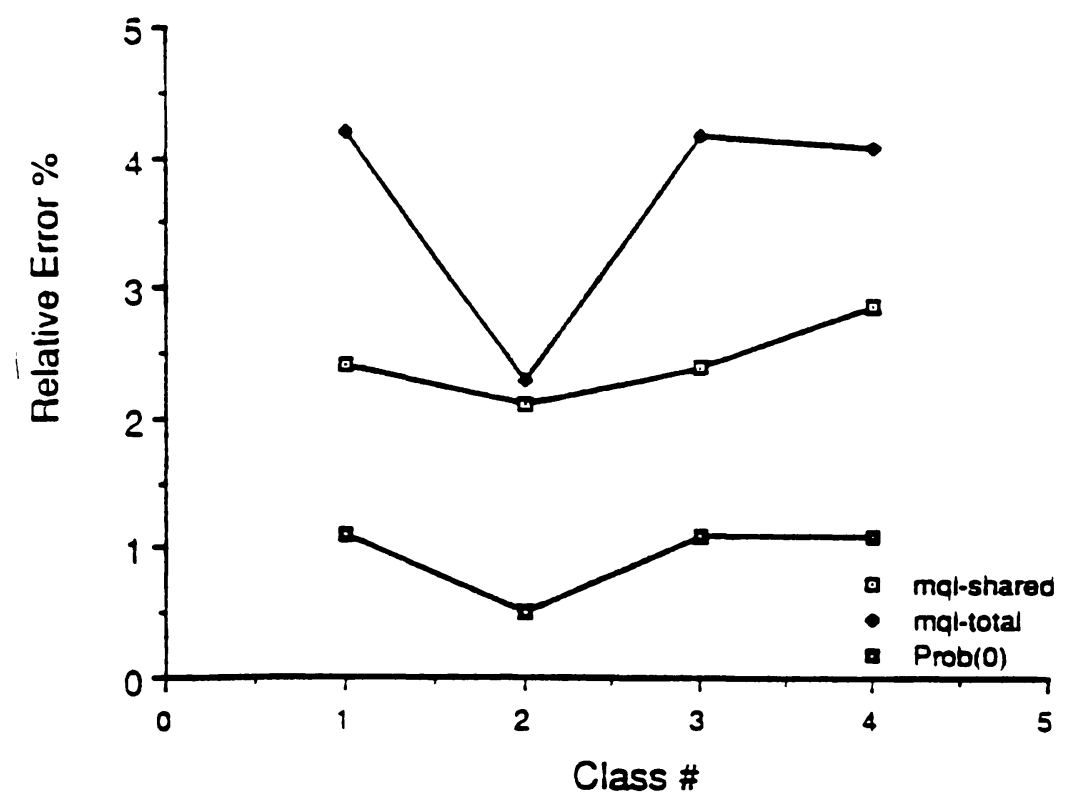

Fig 18: Relative error \% for each class (Example 7 in Table 2)

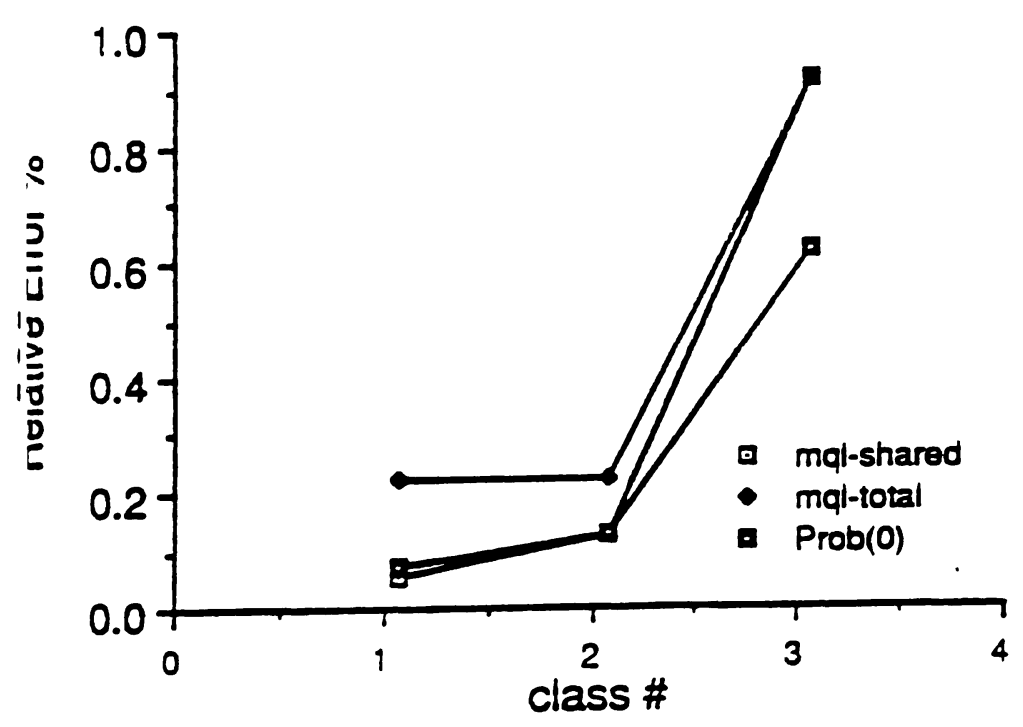

Fig 20: Relative error \% for each class (Example 9 in Table 2)

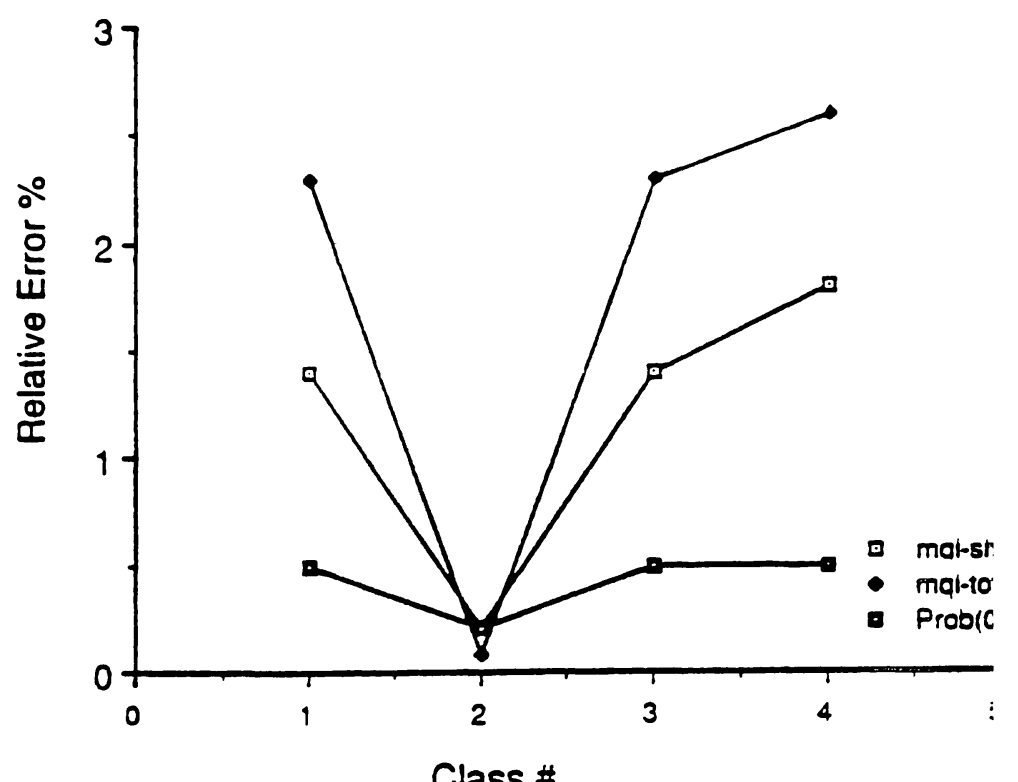

Class \#

Fig 19: Relative error a for each class (Example 8 in Table 2) 


\section{References}

(1) S. Fdida, H.G. Perros and A. Wilks. "Semaphore Queues: Modeling .Multilayered Window Flow Control . Mechanisms". Tech. Rep. Computer Science. 1988. North Carolina State L'niversity

[2] A. Krzesinski and P. Teunissen. ".Multiclass Queueing . Jetworks with Population Constrained Subnetworks". Proc. AC.M SIG.METRICS Conf. on Measurement and Modeling of Computer Systems. Austin. TX (August 1985), 128-139

[3] S.S. Lam, "Queueing . letworks with Population Size Constraints", IBM J. Res. Develp., 370-378, July 1977

[4] E.D. Lazowska and J. Zahorjan, "Multiple Class Memory Constrained Queueing Networks", Proc. ACM SIGMETRICS Conf. on Measurement and Modeling of Computer Systems, Seattle, WA (August 1982), 130-140

[5] M. Reiser, "A Queueing Network Analysis of Computer Communications Network with Window Flow Control", IEEE Trans. Comm., vol. COM 27, 1199-1209, 1979

[6] M. Reiser, "Performance Evaluation of Data Communications Systems", Proc. IEEE, vol. 70, 171-196, 1982

[7] A. Thomasian and P. Bay, "Analysis of Queueing Network Models with Population Size Constraints and Delayed Blocked Customers", Proc. ACM SIGMETRICS Conf., Cambridge, 202-216, 1984 